\title{
Structural studies of dynamic CD4 changes relevant to HIV infection
}

Jennifer Anne Channell ${ }^{1}$, Martine Moulin ${ }^{1}$, Gavin Owen ${ }^{2}$, Nichole Cerutti ${ }^{3}$, Michael Haertlein ${ }^{1}$, Edward Mitchell ${ }^{4}$, Maria Papathanasopoulos ${ }^{2}$, Trevor Forsyth ${ }^{1}$

${ }^{1}$ Life Sciences Group, Institute Laue Langevin, Grenoble, France, ${ }^{2}$ HIV Pathogenesis Research Unit, Wits, Johannesburg, South Africa, ${ }^{3}$ Institute de Biologie Structurale, Grenoble, France, ${ }^{4} E S R F$, Grenoble, France E-mail: channellj@ill.fr

Cluster of differentiation 4 (CD4) plays an important role in the adaptive immune response. It acts co-operatively with the Tcell receptor (TCR) to bind the Major Histocompatibility Complex Class 2 (MHC2) on antigen-presenting cells (APCs) to effect down-stream immune responses. However, it is also the primary receptor for the Human Immunodeficiency Virus-1 (HIV-1) which binds to human CD4+ cells via the surface glycoprotein gp120. CD4 has 4 ectodomains (D1-4) of which domains D1, D2 and D4 contain disulphide bonds. The second domain disulphide bond is classed as an allosteric disulphide, of the configuration "-RHStaple", which has unusually high dihedral energy, resulting in its relatively facile reduction and potential structural realignment.

Biochemical analyses have shown that gp120 can only bind to a monomeric form of CD4 in which its second domain allosteric disulphide bond is reduced $[1,2]$. However, until recently, there has been no biophysical or structural data published that can explain why CD4's redox state is essential for gp120 binding, during HIV-1 infection.

Through a collaboration, between the HIV Pathogenesis Research Unit (HPRU), South Africa, the Insitute Laue-Langevin (ILL), the European Synchrotron (ESRF) and Synthelis, France, biophysical analyses of two-domain CD4 (2dCD4) wild-type and disulphide knock-out mutant proteins has shown that ablation of the allosteric disulphide bond in domain 2 causes an energetically favorable, domain collapse, resulting in increased 2dCD4 stability. Conversely ablation of the structural disulphide bond in domain 1 causes destabilisation of 2dCD4 [3].

Small-angle neutron scattering experiments, exploiting deuterium contrast variation, have allowed low resolution structural analysis of the redox isoform of 2dCD4 bound to gp120. In addition, small-angle X-ray scattering has been used to compare and contrast the size and shape of wild-type 2dCD4 to a panel of 2dCD4 disulphide-bond knock-outs, which mimic the various redox states of 2dCD4-WT.

This novel structural data can help us to understand why gp120 binds this specific, partially reduced form of 2 dCD4 and we anticipate that this information will inform the structure based design of future HIV anti-viral treatments and, hopefully, a prophylactic HIV vaccine.

[1] Matthias, L. J., Azimi, I., Tabrett, C. A. \& Hogg, P. J. Reduced monomeric CD4 is the preferred receptor for HIV.(2010) J. Biol. Chem, 285, pp40793-40799.

[2] Cerutti, N.M., Killick, M., Jugnarain, V., Papathanasopoulos, M. \& Capovilla, A. (2014) Disulfide reduction in CD4 domain 1 or 2 Is essential for interaction with HIV glycoprotein 120 (gp120), which impairs thioredoxin-driven CD4 dimerization. J. Biol. Chem. 289, pp10455-10465

[3] Owen, G.R., Channell, J.A., Forsyth, V.T., Haertlein, M., Mitchell, E., Capovilla, A., Papathanasopoulos, M., and Cerutti, N.M. Human CD4 metastability is a function of the allosteric disulfide bond in domain 2. (2016) Biochemistry. 55(15) pp2227-2237.

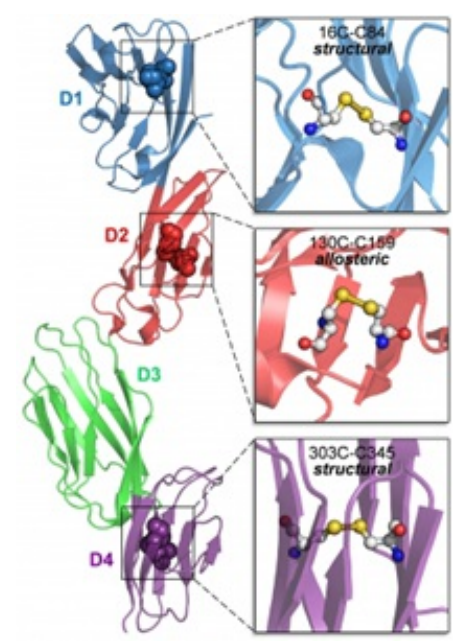

Keywords: $\underline{\text { HIV, neutrons, X-rays }}$ 